\title{
Polysomnography and home documented monitoring of cardiorespiratory pattern
}

\author{
H Daniëls, G Naulaers, F Deroost, H Devlieger
}

\begin{abstract}
Polysomnographic findings were compared with data obtained subsequently from home documented monitoring in order to study the diagnostic value of both techniques. Polysomnography was performed in 1274 infants born prematurely and in 422 patients admitted because of apparent life threatening events (ALTEs). In 72 of the infants, home documented monitoring of the cardiorespiratory pattern, including QRS complexes, was performed. Subsequent documented episodes of heart rate $<50$ beats $/ \mathrm{min}$ were considered as "serious life threatening events". It was confirmed that polysomnographic findings correlated well with subsequent events as registered by home documented monitoring. Especially in the ALTE group where both infants with and without abnormal polysomnography were home monitored, the single polysomnography significantly predicted subsequent life threatening events. Home documented monitoring of the cardiorespiratory pattern has a distinctive advantage over simple cardiorespiratory monitoring as a means to identify and document life threatening events, in addition to its value as a rescue device.

(Arch Dis Child 1999;81:434-436)
\end{abstract}

Keywords: polysomnography; apparent life threatening event; preterm infants; cardiorespiratory home monitoring; sudden infant death syndrome

Polysomnography is a well established, although still controversial, method to identify infants at risk for sudden infant death syndrome (SIDS) who can be subsequently home monitored. ${ }^{1}$ The high frequency of false alarms in the classical event recorder monitors ${ }^{23}$ made it impossible to perform a good evaluation of polysomnography. The development of memory cardiorespiratory monitors offering analogue signals of chest movements and electrocardiogram (ECG), including QRS complexes during alarm episodes, now permits accurate differentiation between true and false alarms.

In this study we compared polysomnoPaediatrics, University Hospital Gasthuisberg, Herestraat 49, B-3000 Leuven, Belgium H Daniëls

G Naulaers

F Deroost

H Devlieger

Correspondence to: Dr Daniëls

Accepted 20 July 1999 STUDY GROUP

Between January 1993 and July 1998 polysomnography was performed in 1274 pre- term infants with a postmenstrual age at birth $<34$ weeks, and in 422 patients diagnosed as having had an apparent life threatening event (ALTE) by the doctor who referred the patient.

METHODS

The polysomnography was performed in the preterm group before discharge at a postmenstrual age of 36-40 weeks, and in the ALTE group as soon as possible after the event at a postnatal age varying from $0-16$ weeks.

All patients admitted because of ALTE underwent a clinical examination; peripheral blood glucose and ammonia concentrations, and electrolytes, were measured, and renal and liver function tests, urinalysis, and chest radiography were performed. When there was a history of gastro-oesophageal reflux, a barium oesophagram was performed to rule out anatomical lesions. Following re-examination, no new diagnosis was made in these patients. ${ }^{4}$

Polysomnography was carried out for a minimum of 8 hours and a maximum of 12 hours. The nine channels registered included ECG, ECG derived beat to beat heart rate, thoracic and abdominal breathing movements, nasal air flow as measured by means of a nasal thermistor, chin electromyogram to detect nutritive and non-nutritive sucking, ${ }^{56}$ electrooculogram to detect sleep states, and electroencephalogram to exclude epileptic activity. A computer based oscillograph and data acquisition system (CODAS, Dataq Instruments, Akron Ohio, USA) was adapted in our unit for acquisition, evaluation, and reproduction of the polysomnographic recordings.

All the polysomnographic data were examined and evaluated manually by a single observer ( $\mathrm{HDa})$. The criteria for normality were no central or obstructive apnoea for more then $15 \mathrm{~s}$, no bradycardia below 60 beats per minutes (bpm) for $>3 \mathrm{~s}$, and no oxygen desaturation below $80 \%$ for $>5 \mathrm{~s}$.

Home documented monitoring of the cardiorespiratory pattern, including QRS complexes, was initiated when polysomnography revealed (outside the feeding period) life threatening events, defined as bradycardia $(<50 \mathrm{bpm}$ for minimum $4 \mathrm{~s}$ ) and/or severe obstructive apnoea for more than $15 \mathrm{~s}$ with bradycardia below $60 \mathrm{bpm}$, with oxygen desaturation $(<80 \%)$. In addition, home documented monitoring of the cardiorespiratory pattern was initiated in infants with "severe" ALTE, as defined by the National Institutes of Health (NIH) consensus, ${ }^{8}$ when polysomnography was normal.

We did not consider prolonged central apnoea for more than $15 \mathrm{~s}$ as an indicator for using home documented monitors; instead 

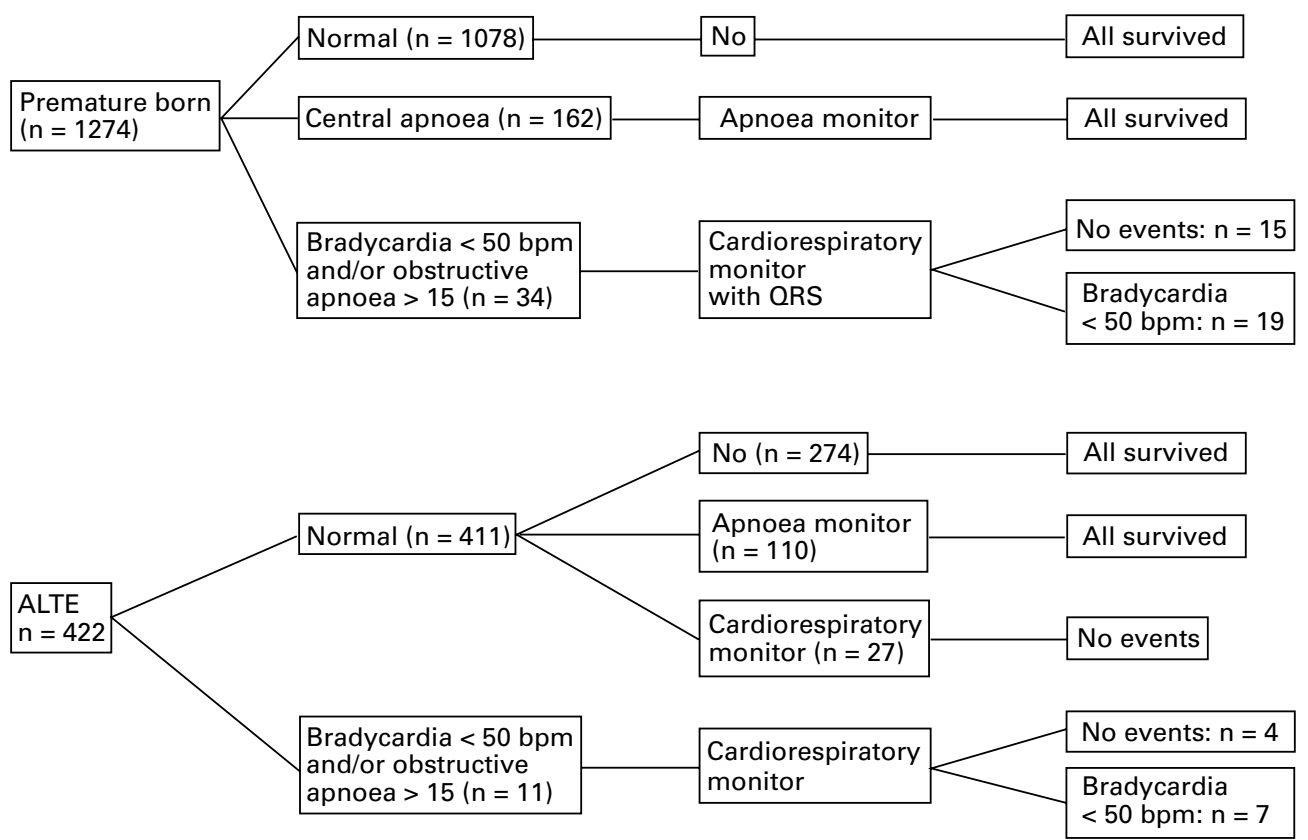

Figure 1 Flow diagram showing aetiology, results of the polysomnography, treatment, and follow up.

apnoea monitors were used. Apnoea monitors were also used when the ALTE was not considered as "severe" because no resuscitation was required. Since no accurate differentiation of true alarms or false alarms is possible using apnoea monitors, we will not consider these infants in this article.

The memory cardiorespiratory monitor systems used were the Arvee 4800 (Arvee Medical, Austen, Texas, USA) and the Nellcor Edentec 336 assurance monitor (Nellcor Puritan Bennett, Eden Prairie, Minnesota, USA). Both types of monitors are able to memorise ECG trends, QRS complexes, and respiratory waveforms and could also be used in connection with a pulse oximeter. The alarm for apnoea was set at $20 \mathrm{~s}$. The bradycardia threshold was $60 \mathrm{bpm}$. Only episodes of heart rate $<50 \mathrm{bpm}$ were considered as "serious" events because Hunt et al never recorded bardycardia $<50 \mathrm{bpm}$ in normal infants. ${ }^{9}$ The

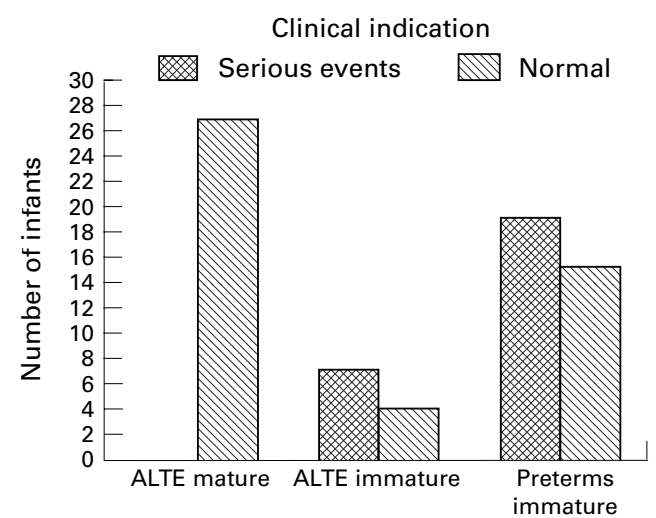

Figure 2 Results of home documented monitoring of cardiorespiratory pattern, according to clinical indication. The ALTE group is divided into infants with mature and immature cardiorespiratory control during polysomnography. duration of this type of home monitoring varied from a minimum of two months to a maximum of 18 months (median five months).

Comparison between groups was done using the $\chi^{2}$ test or Fisher's exact probability test. Significance was set at $\mathrm{p}<0.05$ one tailed for the $\chi^{2}$ test and $p<0.05$ two tailed for the Fisher's exact test. ${ }^{10}$

\section{Results}

During the study period 72 infants received home documented monitoring of the cardiorespiratory pattern with QRS complex. These patients were distributed into two groups: one group of 34 former premature infants and 11 infants with ALTE, who had life threatening events on polysomnography, and a second group of 27 patients who had "severe" ALTE as defined by the NIH consensus. ${ }^{8}$ Further diagnostic evaluations, as mentioned above, were negative. Twenty six of the 45 infants with life threatening events on polysomnography had "real" alarms as judged by the investigator on the basis of the stored data and the reports of the parents concerning resuscitation of the infant. The relation between abnormal polysomnography and the home documented monitoring of the cardiorespiratory patterns in the whole group of 72 infants was $p<0.001$ as calculated by the $\chi^{2}$ test.

As shown in the flow diagram (fig 1) seven of the 11 infants with an ALTE and serious life threatening events on polysomnography had subsequent life threatening events, as documented by home monitoring of the cardiorespiratory pattern, with the lowest heart rate varying from 16-35 bpm. These events are thought to be caused by obstructive apnoea, although this cannot be identified by impedance monitors. ${ }^{11}$ None of the 27 infants with ALTE and a normal polysomnography had 
subsequent life threatening events (Fisher's exact two tailed $\mathrm{p}<0.001$ ).

As shown in fig 2, 19 out of 34 preterm infants with immature cardiorespiratory patterns during polysomnography had subsequent documented life threatening events caused by central or obstructive apnoea, with bradycardia varying from $12-41 \mathrm{bpm}$.

\section{Discussion}

Despite the controversy over polysomnography, which has been the subject of considerable comment following the Hoyt conviction, ${ }^{12}$ this study shows that polysomnography can identify infants surviving an ALTE, who are at risk of severe life threatening events. None of the infants with a normal polysomnography had a subsequent event while seven of 11 who had an abnormal polysomnography did. Although the number is small, this shows the predictive value of polysomnography in infants surviving an ALTE. This was also described by Côté and colleagues. ${ }^{13}$

The value of polysomnography in identifying infants at risk can be extended to the group of infants born prematurely. Although none of the infants with normal polysomnography were monitored at home, close follow up showed that none of them had a serious event or died from SIDS. This suggests that preterm infants at increased risk of subsequent life threatening events can be identified by polysomnography by the end of their stay in the neonatal intensive care unit, when they reach 35-38 weeks of gestational age, as reported by Côté and colleagues ${ }^{13}$ and by Brooks. ${ }^{14}$ Even for infants with immature cardiorespiratory control early discharge becomes possible providing a cardiorespiratory monitor is supplied and appropriate instructions are given to the parents. Prolonged stay on the intensive care unit only for the reason of immature cardiorespiratory control can be prevented by this strategy. However, this needs to be confirmed by longer studies.

If polysomnography shows a life threatening event, home documented monitoring of the cardiorespiratory pattern, including QRS complex, is preferable to respiratory and cardiorespiratory monitors without a memory. Respiratory monitors are less accurate since the controversy surrounding the putative relation between apnoea without bradycardia and SIDS. ${ }^{12}$ Accurate differentiation of true apnoea and true bradycardia events from false alarms is only possible using home documented monitoring with QRS complex, avoiding prolonged home monitoring caused by false alarms and subsequent anxiety of the parents. This, and the fact that consecutive expensive hospitalisations are no longer necessary, highly compensates the additional cost of home documented cardiorespiratory monitors. Hunt $^{15}$ and Steinschneider and colleagues ${ }^{16}$ also came to the conclusion that, despite the increased monthly cost, home monitoring incorporating event recording resulted in a lower average cost per patient and needed to become the standard.

In conclusion, in 26 of the 45 infants (58\%) with abnormal polysomnography, life threatening events were detected by home documented monitoring of the cardiorespiratory pattern, using the criteria of bradycardia $<50 \mathrm{bpm}$, while bradycardia $<50 \mathrm{bpm}$ was never recorded in infants at risk with normal polysomnography. The identification of infants at risk for SIDS, such as survivors of ALTE and preterm infants with immature cardiorespiratory control, by means of polysomnography, and the follow up of infants at risk by memory cardiorespiratory home monitoring has proven to be a safe and cost effective strategy. Further studies to confirm this validation are necessary.

1 Bentele KHP, Albani M. Are there tests predictive for prolonged apnoea and SIDS? Acta Paediatr Scand 1988;77(suppl):342

2 Weese-Mayer DE, Brouillette RT, Morrow AS, et al. Assessing validity of infant monitor alarms with event recording. f Pediatr 1989;115:702-8.

3 Steinschneider A, Santos V. Parental reports of apnea and bradycardia: temporal characteristics and accuracy. Pediatrics 1991;88:1100-5.

4 Samuels MP, Poets CF, Noyes JP, et al. Diagnosis and management after life threatening events in infants and young children who receive cardiopulmonary resuscitation. BMF 1993;306:489-92.

5 Daniëls H, Devlieger H, Casaer P, et al. Nutritive and nonnutritive sucking in preterm infants. $\mathcal{F}$ Dev Physiol 1986;8: $117-21$

6 Daniëls H, Devlieger H, Minami T, et al. Infant feeding and cardiorespiratory maturation. Neuropediatrics 1990;21:910.

7 Devlieger H, Daniëls H, Deroost F, et al. Assessment of cardiorespiratory stability of infants to be discharged from the NICU. Evaluation of a new data processing system. F Perinat Med 1989;17(suppl I):101.

8 National Institutes of Health. Consensus statement on infantile apnea and home monitoring. Pediatrics 1987;79: 292-9.

9 Hunt CE, Hufford DR, Bourguignon C, et al. Home documented monitoring of cardiorespiratory pattern and oxygen saturation in healthy infants. Pediatr Res 1996;39: 216-22.

10 Siegel S. Nonparametric statistics for the behavioral sciences. Tokyo: McGraw-Hill Kogakusha, 1956.

11 Brouillette RT, Morrow AS, Weese-Mayer DE, et al. Comparison of respiratory inductive plethysmography and thoracic impedance monitor for apnea monitoring. f Pediatr 1987;111:377-83.

12 Bergman AB. Wrong turns in sudden infant death syndrome research. Pediatrics 1997;99:119-21.

13 Côté A, Hum C, Brouillette RT, et al. Frequency and timing of recurrent events in infants using home cardiorespiratory monitors. F Pediatr 1998;312:783-98.

14 Brooks JG. Infantile apnea and home monitoring. Pediatrician 1988;15:212-16.

5 Hunt CE. Cardiorespiratory monitoring. Clin Perinatol 1991;18:473-95.

16 Steinschneider A, Santos V, Freed G. Cost implications of event recordings in apnea/bradycardia home monitoring: a theoretical analysis. Pediatrics 1995;95:378-80. 